UCRL-JC-130922

PREPRINT

\title{
Measurements of the Lick Observatory Sodium Laser Guide Star
}

\author{
Donald T. Gavel \\ Herbert W. Friedman
}

This paper was prepared for submittal to the SPIE 1998 Symposium on Astronomical Telescopes and Instrumentation Kona, $\mathrm{HI}$

March 20-28, 1998

March 1998

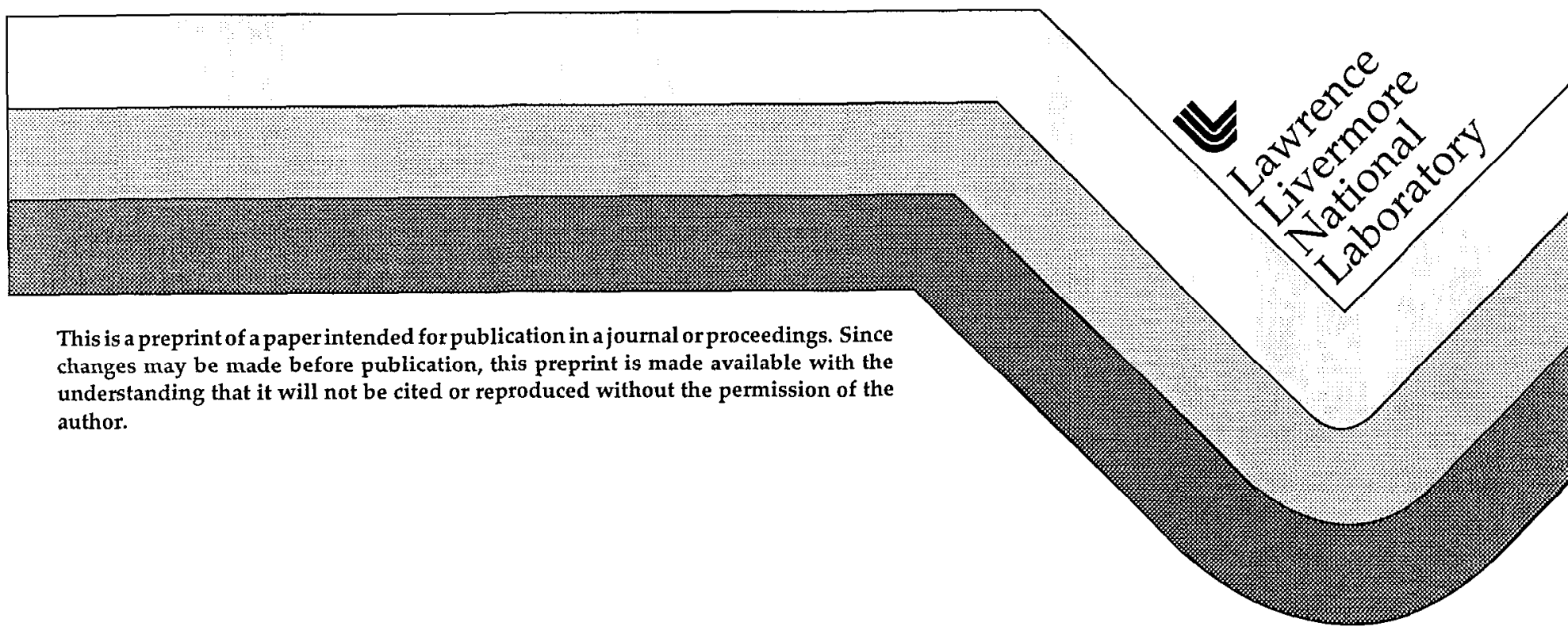




\section{DISCLAIMER}

This document was prepared as an account of work sponsored by an agency of the United States Government. Neither the United States Government nor the University of California nor any of their employees, makes any warranty, express or implied, or assumes any legal liability or responsibility for the accuracy, completeness, or usefulness of any information, apparatus, product, or process

disclosed, or represents that its use would not infringe privately owned rights. Reference herein to any specific commercial product, process, or service by trade name, trademark, manufacturer, or otherwise, does not necessarily constitute or imply its endorsement, recommendation, or favoring by the United States Government or the University of California. The views and opinions of authors expressed herein do not necessarily state or reflect those of the United States Government or the University of California, and shall not be used for advertising or product endorsement purposes. 


\title{
Measurements of the Lick Observatory sodium laser guide star
}

\author{
Donald T. Gavel ${ }^{1}$, Herbert W. Friedman \\ Lawrence Livermore National Laboratory
}

\begin{abstract}
The Lick Observatory guide star laser has provided a beacon sufficient to close the adaptive optics loop and produce corrected images during runs in 1996 and 1997. This report summarizes measurements of the wavefront quality of the outgoing beam, photoreturn signal from the sodium beacon, and radiancc distribution of the guide star on the sky, and follows with an analysis of the impact of the laser on adaptive optics system performance.
\end{abstract}

\section{Introduction}

Corrected images using laser guide star based adaptive optics system have recently been demonstrated at Lick Observatory [1]. We have performed a number of measurements of the laser beam and the resulting guide star in order to characterize its brightness and intensity distribution

The Lick system is outfitted with a number of diagnostics to observe both near and far field characteristics of the laser. Also, by changing a dichroic in the AO system, we can obtain high resolution images of the guide star spot in the sodium layer. Here, we present the results of guide star measurements taken over the past two years, with an analysis of the guidestar's characteristics on the performance of the AO system. Some straightforward modifications to the laser system are currently planned that should improve the beam quality for this year's AO operations.

\section{The Lick sodium guide star laser and diagnostics system}

The Lick laser is a 20 Watts tuned dye laser having a pulse repetition frequency of $11 \mathrm{kHz}$ and a pulse width of $150 \mathrm{~ns}$ that operates at a frequency in the middle of the sodium $\mathrm{D}_{2}$ line at 0.589 microns. The laser is electro-optically phase modulated to broaden it to $1.5 \mathrm{GHz}$ line width, producing a spectrum that attempts to address all of the atoms in the Doppler-broadened absorption line of mesospheric sodium, and thereby avoiding saturation effects due to the high peak power of the pulsed laser. We refer the reader to earlier work [2] for more details of this laser system.

The laser light is projected from a $30 \mathrm{~cm}$ diameter projection optic located off the side of the Shane 3 metcr telescope, with 1 meter separation betwecn the output optic and edge of the Shane aperture (Figure 1). The beam itself is square, the shape of the last dye amplifier channel, and has been measured at $14 \mathrm{~cm}$ on a side. This is somewhat smaller than can actually be accommodated by the last optic. The laser system contains a diagnostic package that samples some of the outgoing laser light for near and far-field quality, as well as establishes pointing and centering control. The near and far field intensity images are recorded with standard 8-bit digital cameras, and the wavefront phase is measured with a Hartmann sensor.

\footnotetext{
${ }^{1}$ Address correspondence to: Donald T. Gavel, LLNL, L-458, P.O. Box 808, Livermore, CA, 94550. E-mail: gavel1@1lnl.gov
} 

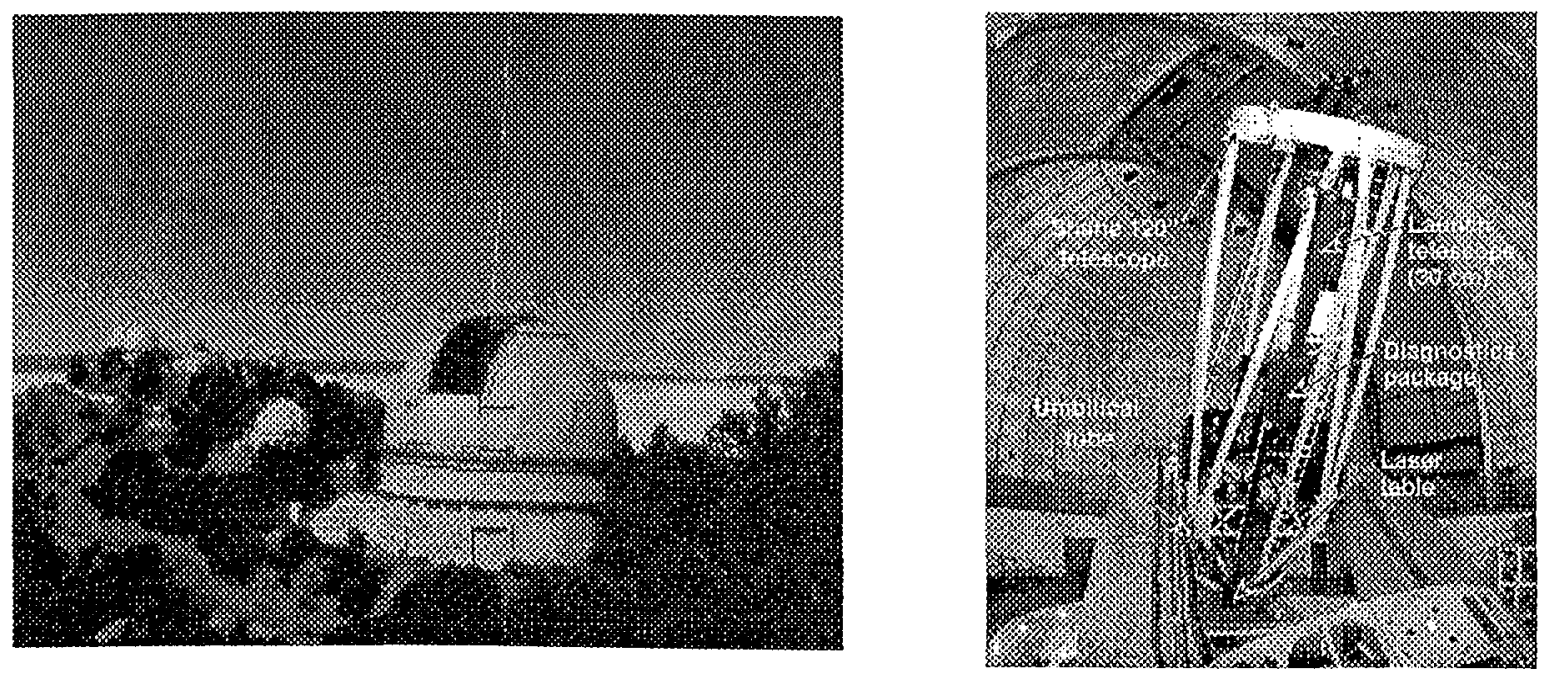

Figure 1 Left: Laser beam exiting the Shane telescope dome. Right: Laser mounted on the side of the 3 meter telescope.

\section{Guide star return signal}

Figure 2 shows measurements of laser guide star return flux from 1996 and 1997 laser guide star runs. The return flux shows the same variability with season that was observed in earlier lidar measurements of sodium abundance by Gardner [3]. The AO system control loop had enough signal to lock at $55 \mathrm{~Hz}$ sample rate in the periods of low sodium (May and July), and at $200 \mathrm{~Hz}$ in periods of high sodium (September, October, and November). Improved images of astronomical objects have been obtained only in the high seasons.

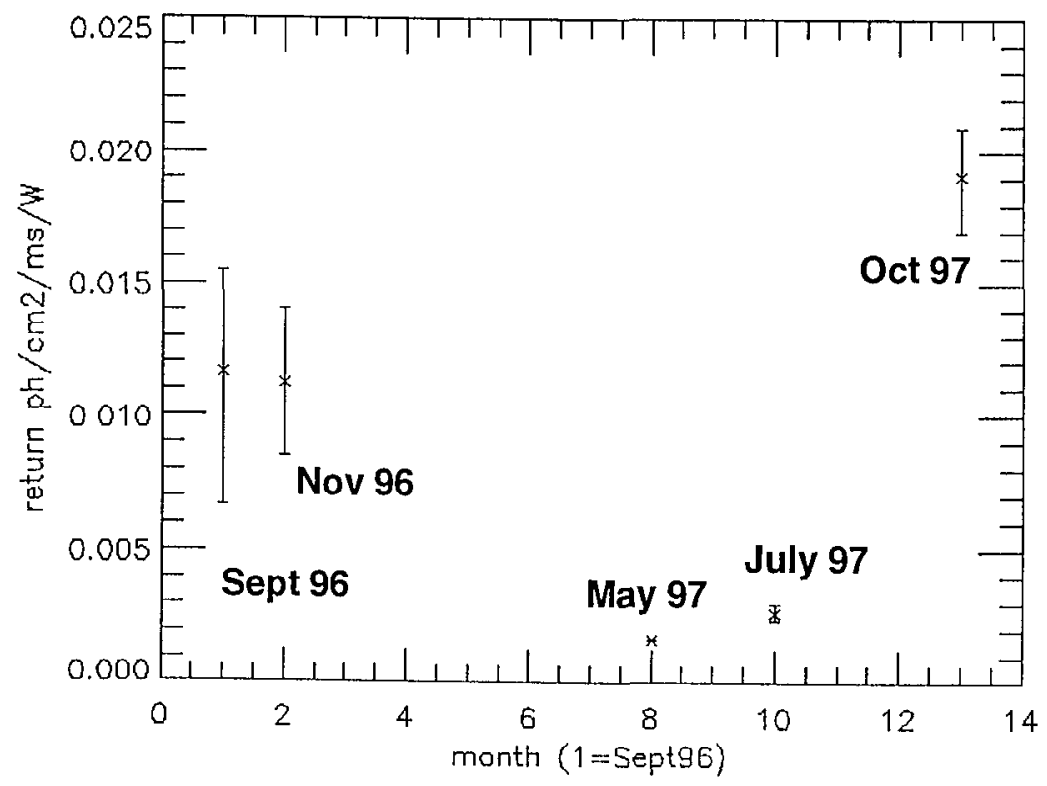

Figure 2 Normalized laser guidestar return signal over a two year campaign. Bars indicate variation night to night during a run. After accounting for system throughput, and assuming a linear sodium response model, the return signal of 0.015 photons $/ \mathrm{ms} / \mathrm{cm}^{2} /$ Watt corresponds to a sodium column density of $5 \times 10^{9} / \mathrm{cm}^{2}$. 


\section{Outgoing beam quality}

Near and far-field images of the outgoing beam are shown in Figure 3. With the power amplifier turned off (preamp power level) the beam is fairly uniform, and the far field pattern resembles that of a diffraction-limited square beam pattern. The distance from the center to the first zero in the diffraction pattern is 0.87 arcseconds on the sky.

With the laser amplitier operating at 7 Watts, the far field shows some blurring of the central lobe and with some energy entering the next order, particularly in the $\mathrm{x}$ direction (this is the direction of the pump light in the amplifier). There is also light beginning to appear in a halo about 4 arcseconds in radius. (Note that the log scale exaggerates the low level intensities). At this power level, roughly $35 \%$ of the energy of the laser is within the central core of the far field spot. This is to be compared with $83 \%$ theoretical maximum in the diffraction-limited case.

At 18 Watts output power, the far field spot shows considerable light in the halo, with spot widening favoring the $x$ direction. At this power level, 25 to $30 \%$ of the energy is in the central core. The near field beam shows a slight change to a trapezoidal shape, possibly due to non-uniform thermally induced focusing in the amplifier dye cell.

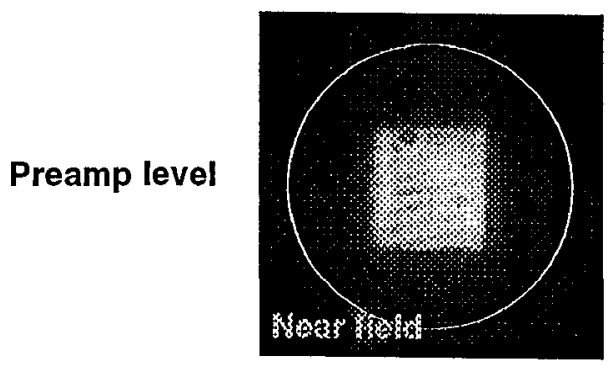

7 Watts
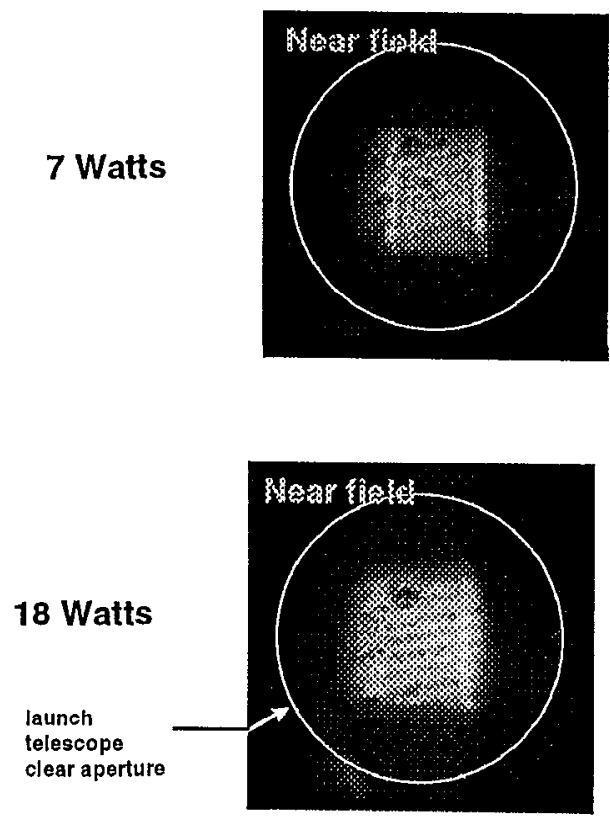

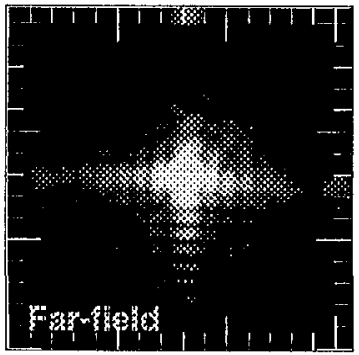

(log stretch, clipped)

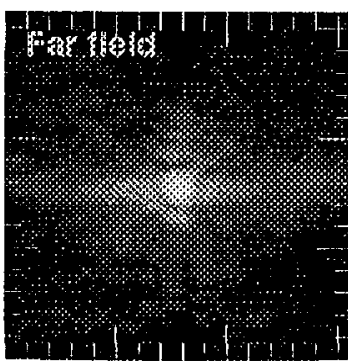

(log stretch)

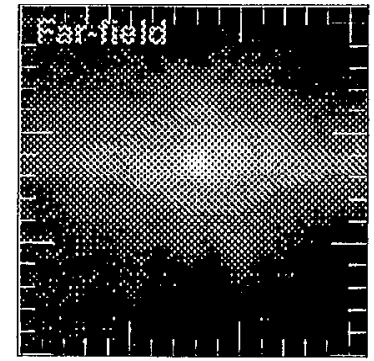

(log stretch)

Figure 3 Near and far-field images of the exiting laser beam. Tick marks on the farfield images represent 1 arcsecond intervals on the sky. The circle on the near-field image indicates the $30 \mathrm{~cm}$ diameter of the final optic of the launch telescope 


\section{Images of the guidestar on the sky}

One night in October, 1997 was dedicated to observing the laser guide star spot with the receiving telescope. With the dichroic splitter removed from the AO system, the guide star light travels down the science path of the system. A Photometrix visible light $\mathrm{CCD}$ was used to take long exposure images at 0.05 arcsecond resolution.

Oct. 17, 1997, $P_{\text {lasor }}=7.5$ Watts, 1 arcsec seeing, 10 sec. exposure
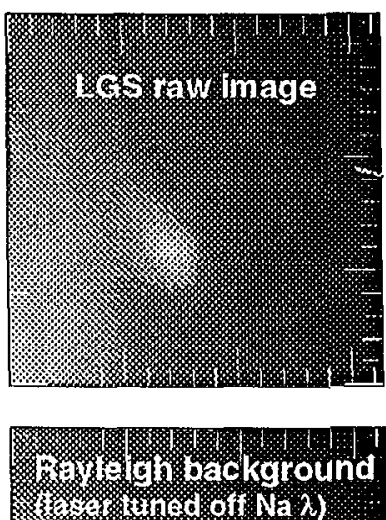

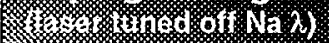

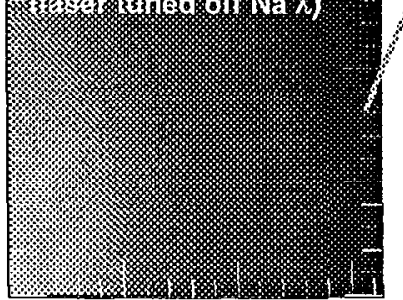

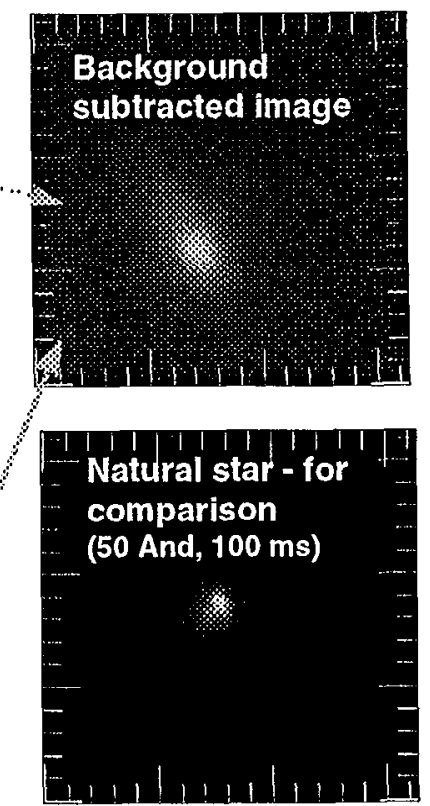

Figure 4 Images of the guide star and a natural star taken with the receiving camera (Photometrix CCD, 0.05 arcseconds/pixel plate scale). The tick marks indicate 1 arcsecond intervals on the sky.

We compare the encircled energies for the outgoing laser profile to that of the observed LGS spot in Figure 5. The energy curve of the 7.5 Watt laser guide star is in approximate agreement with the 7 Watt outgoing far-field spot convolved twice (once for upgoing path, once for downgoing path) with the atmospheric PSF.

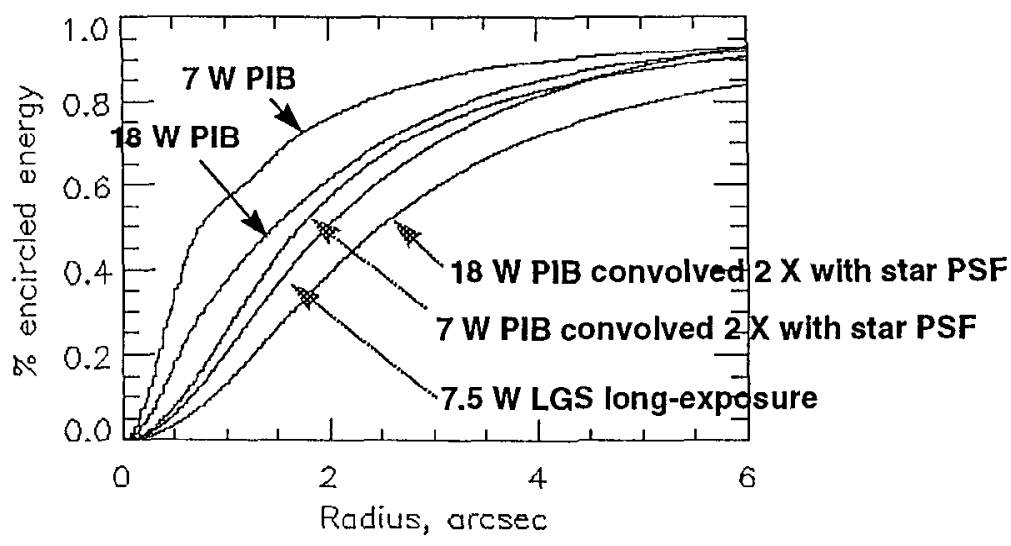

Figure 5 Encircled energy of the outgoing laser far field images (labeled PIB) compared with the long exposure image of the laser guidestar with the receiving telescope (LGS long-exposure). 


\section{Impact on system performance}

Wavefront error depends on guide star spot intensity distribution according to:

$$
\sigma_{\mathrm{SNR}}=\chi d \frac{\int_{\infty}^{\infty} d y \int_{\infty}^{\infty} d x f(x, y)}{2 \int_{-\infty}^{\infty} d y f(0, y)} \times \frac{1}{\mathrm{SNR}}
$$

where $f(x, y)$ is the distribution of light on the detector ( $x$ and $y$ given in units of angle on the sky), SNR is the signal to noise ratio on the detector, $d$ is the Hartmann subaperture size, and $\chi$ is a factor that accounts for AO control loop averaging. The factor in the middle, the ratio of integrals of the light distribution, is a measure of the spot size which has units of angle on the sky. The wavefront error (which is in distance units, depends linearly on the spot size factor and inversely on the brightness (through the signal-to-noise ratio).

There can be significant Strehl improvement by making the spot smaller, as shown in Table 1 where we calculate the spot size factor and error contribution for several guide star cases. A natural star has the smallest spot size error contribution. The measured far-field laser spot at each laser power was convolved with the seeing (star) to get an estimate of the contribution from laser guide star spot size. In the worst case at the 18 Watts level, the laser guidestar spot size factor is almost a factor of 3 larger than that of the natural star. A perfect laser guide star, one with a diffraction-limited square-beam far-field pattern, will have a spot size factor much closer to the ideal natural star case.

Table 1 Wavefront error contribution for several guide star cases

\begin{tabular}{|l|c|c|c|c|}
\hline & Spot Size Factor & SNR & $\sigma_{\text {SNR }}$ & $\begin{array}{c}\text { Strehl Contribution } \\
\text { at } \lambda=2 \mu \mathrm{m}\end{array}$ \\
\hline Star & 1.05 arcsec & 20.8 & $123 \mathrm{~nm}$ & 0.86 \\
\hline LGS 18 watts * star & 2.97 arcsec & 20.8 & $340 \mathrm{~nm}$ & 0.32 \\
\hline LGS 7 Watts * star & 1.74 arcsec & 15.5 & $268 \mathrm{~nm}$ & 0.49 \\
\hline $\begin{array}{l}\text { "Perfect" 18 Watt } \\
\text { LGS * star }\end{array}$ & 1.28 arcsec & 20.8 & $149 \mathrm{~nm}$ & 0.80 \\
\hline
\end{tabular}

The wavefront error contributions shown in Table 1 should be compared with the other dominant sources of wavefront error in the Lick AO system: the fitting error (due to the finite number of actuators on the deformable mirror), which is $130 \mathrm{~nm} \mathrm{rms}$, and the servo following error which is $180 \mathrm{~nm} \mathrm{rms}$ (assuming a sampling frequency of $\mathrm{fs}=200 \mathrm{~Hz}$, and a Greenwood frequency of $\mathrm{fg}=100 \mathrm{~Hz}$ ).

\section{Planned improvements to the Lick laser}

Improvements to wavefront quality will result in a smaller guide star spot with reduccd halo, thercby decreasing the wavefront measurement error term. Our plans are to incorporate what we have learned in developing a second-generation sodium guidestar laser (to be delivered to the Keck Observatory) back into the Lick system. In particular, wavefront quality will be significantly improved by implementing a better method of sending the light through the dye amplifiers. In the new configuration, the beam is reflected once off a wall of the amplifier dye cell rather than going straight through, with the result being that dye medium induced wavefront aberrations tend to average to zero. This geometry also allows a round instead of square beam to pass through the (rectangular shaped) dye cell, which gives a better fill factor on the final projecting optic.

Initial measurements of the Keck laser in the laboratory are quite encouraging. Enclosed energy fraction in the central lobe remained above $70 \%$ (compared to $30 \%$ for Lick and $83 \%$ for theoretical diffractionlimited) at full power for the duration of an 8 hour hands-off test run (Figure 6).

The straightforward conversion of the Lick laser to bounce-beam will take place before this summer's runs. 

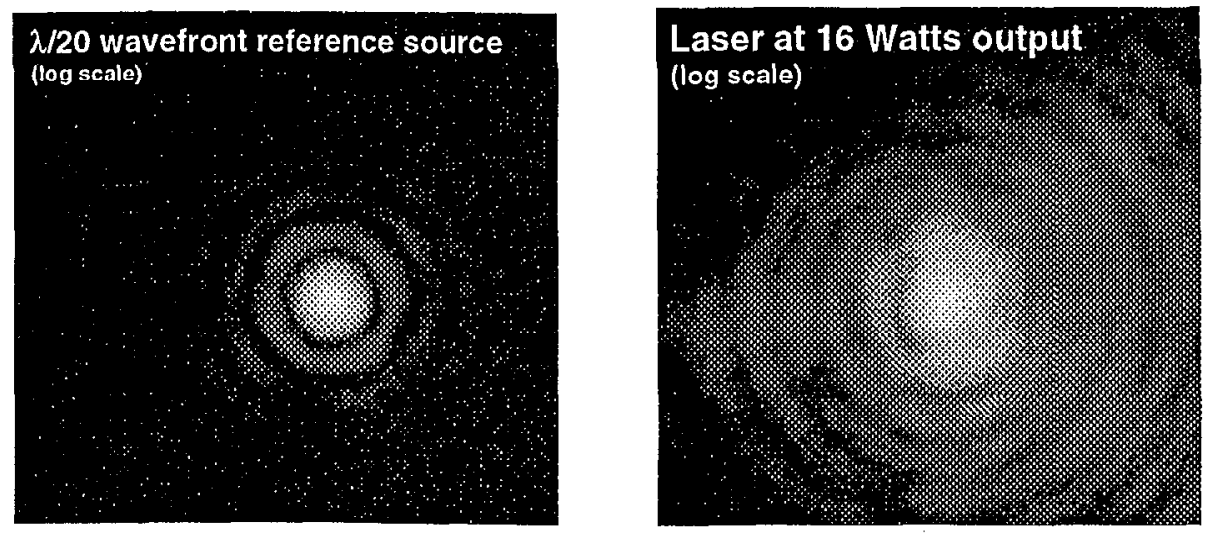

Figure 6 Laboratory test results from the Keck sodium guidestar laser. The far-field beam pattern has greater than $70 \%$ of the laser energy in the central diffraction-limited core.

\section{Conclusions}

During our observations in 1996-1997, we obscrved large seasonal variation in sodium guidestar return. The seasonal variation agrees well with sodium abundance variations observed by Gardner et. al. The Lick laser guide star system has operated closed loop at $55 \mathrm{~Hz}$ sample ratc during the low season, and at $200 \mathrm{~Hz}$ in the high season. Improved images have been achieved only in the high season.

The Lick laser presently produces a $\theta_{50}=4$ arcsecond, $\theta_{80}=8$ arcsecond spot at 18 watts output power in 1 arcsecond seeing. $30 \%$ of the outgoing energy is in the diffraction-limited core ( 0.87 arcsecond radius). This yields a $340 \mathrm{~nm}$ rms wavefront measurement error contribution (compare to $130 \mathrm{~nm}$ of deformable mirror wavefront fitting error).

The new bounce-beam amplifier configuration should increase effective spot radiance by a factor of 2 . This is due to both better beam quality and a larger, round instead of square, beam. This should result in a $150 \mathrm{~nm} \mathrm{rms}$ wavefront measurement error contribution. Initial results from tests with the Keck laser at high power have demonstrated the ability to put $71 \%$ of the energy in the central core.

\section{References}

1. Max, C. E., et. al., Science, Vol 277, No 5332, 1649-1652, Sept 12, 1997.

2. Friedman, H.W., et. al., SPIE, Vol 2534, 150-160, 1995.

3. Gardner, Welsh, and Thompson, Proceedings of the IEEE, v78, 11, 1990.

\section{Acknowledgment}

This work was performed under the auspices of the U. S. Department of Energy by the Lawrence Livermore National Laboratory under contract number W-7405-ENG-48. It was supported by Laboratory Directed Research and Development funding within the University Relations Program and the Lasers Directorate. This support is gratefully acknowledged. 


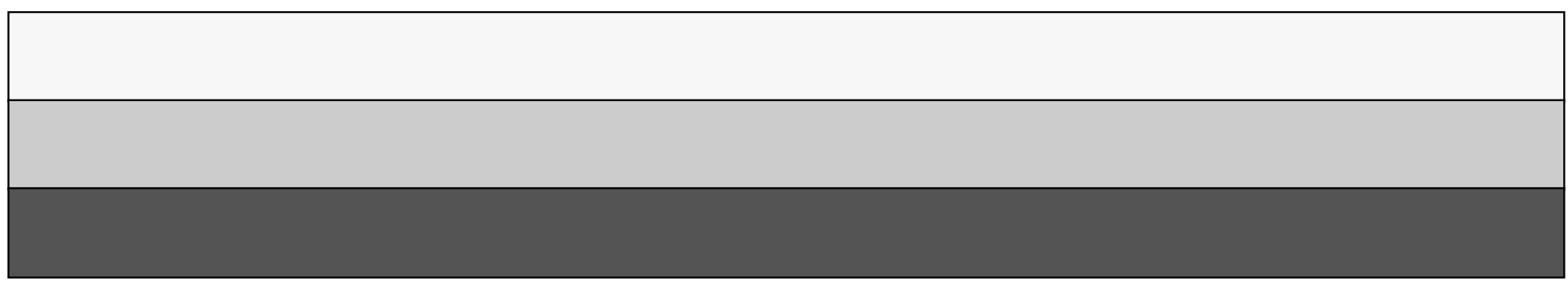

